\title{
Variation in sequences containing microsatellite motifs in the perennial biomass and forage grass, Phalaris arundinacea (Poaceae)
}

\author{
Susanne Barth ${ }^{1 *} \mathbb{0}$, Marta Jolanta Jankowska ${ }^{1}$, Trevor Roland Hodkinson² ${ }^{2}$ Tia Vellani ${ }^{1}$ and Manfred Klaas ${ }^{1}$
}

\begin{abstract}
Forty three microsatellite markers were developed for further genetic characterisation of a forage and biomass grass crop, for which genomic resources are currently scarce. The microsatellite markers were developed from a normalized EST-SSR library. All of the 43 markers gave a clear banding pattern on $3 \%$ Metaphor agarose gels. Eight selected SSR markers were tested in detail for polymorphism across eleven DNA samples of large geographic distribution across Europe. The new set of 43 SSR markers will help future research to characterise the genetic structure and diversity of Phalaris arundinacea, with a potential to further understand its invasive character in North American wetlands, as well as aid in breeding work for desired biomass and forage traits. P. arundinacea is particularly valued in the northern latitude as a crop with high biomass potential, even more so on marginal lands.
\end{abstract}

Keywords: Poaceae, Microsatellite markers, Phalaris arundinacea, Reed canary grass, SSR

\section{Introduction}

Slight changes in the genetic code, such as single nucleotide polymorphisms (SNPs) and single sequence repeats (SSRs) can be directly linked to phenotype differences. Hence, the development and characterisation of novel genetic markers can be of great help to breeders. SSRs have been commonly applied in quantifying genetic variation and analysing the gene flow and parentage in plants [1]. Some recent applications also include hybrid identification [2]. Single sequence repeats are abundant in the genome, multi-allelic and polymorphic and often can be cross-amplified on related species [3]. Next-generation sequencing can provide large numbers of SSRs as demonstrated in this study and is even more useful once converted into routinely applicable genetic markers.

The genus Phalaris belongs to the tribe Aveneae of the subfamily Pooideae of the grass and cereal family Poaceae [4]. Reed canarygrass (Phalaris arundinacea L.) is a tall, perennial C3 grass which is distributed throughout

\footnotetext{
*Correspondence: susanne.barth@teagasc.ie

${ }^{1}$ Teagasc Crops Environment and Land Use Programme, Oak Park Research Centre, Carlow, Ireland

Full list of author information is available at the end of the article
}

Europe and in temperate regions of North America and Asia $[5,6]$. On many sites it forms dense monospecific stands [7]. Considered an invasive wetland species [8, 9], $P$. arundinacea has been successfully introduced into nearly all continents except Antarctica. It is most commonly found growing along water margins and as such has been long recognised as a crop with a high biomass potential, particularly on marginal lands. Reed canarygrass, although not as productive as other grasses (i.e., Panicum virgatum) presents a unique set of characters that make it particularly tolerant to Northern climates [10]. Its high genetic variability has been observed in differences in production rates [8], forage yields [11] and photosynthetic characteristics [12] among others. Other non-crop uses of reed canarygrass include phytoremediation [13], erosion control [14] and paper production [15]. The newly developed primers presented in this publication were tested across a wide range of environmentally and climatically different regions from six European countries of Northern European distribution (Table 1). A subsample of three reactions per primer with one exception (primer TeaPh_nSSR_25) were chosen for sequencing (Fig. 1). These microsatellites can potentially be used for fingerprinting, GenBank accession characterisation 
Table 1 Eleven genotypes characterized in this study

\begin{tabular}{llll}
\hline Country & Sample name & Latitude & Longitude \\
\hline Poland & $\mathrm{A}$ & $53^{\circ} 50^{\prime} 02.83^{\prime \prime} \mathrm{N}$ & $21^{\circ} 03^{\prime} 30.36^{\prime \prime} \mathrm{E}$ \\
Poland & $\mathrm{H}$ & $54^{\circ} 23^{\prime} 21.42^{\prime \prime} \mathrm{N}$ & $18^{\circ} 28^{\prime} 42.18^{\prime \prime} \mathrm{E}$ \\
Germany & $\mathrm{B}$ & $52^{\circ} 13^{\prime} 09.87^{\prime \prime} \mathrm{N}$ & $11^{\circ} 42^{\prime} 25.14^{\prime \prime} \mathrm{E}$ \\
Germany & $\mathrm{D}$ & $53^{\circ} 25^{\prime} 51.60^{\prime \prime} \mathrm{N}$ & $09^{\circ} 46^{\prime} 39.78^{\prime \prime} \mathrm{E}$ \\
Denmark & I & $56^{\circ} 12^{\prime} 13.48^{\prime \prime} \mathrm{N}$ & $08^{\circ} 09^{\prime} 39.07^{\prime \prime} \mathrm{E}$ \\
Denmark & $\mathrm{E}$ & $55^{\circ} 56^{\prime} 16.98^{\prime \prime} \mathrm{N}$ & $12^{\circ} 28^{\prime} 35.70^{\prime \prime} \mathrm{E}$ \\
Sweden & $\mathrm{F}$ & $58^{\circ} 52^{\prime} 20.04^{\prime \prime} \mathrm{N}$ & $14^{\circ} 53^{\prime} 56.79^{\prime \prime} \mathrm{E}$ \\
Sweden & $\mathrm{K}$ & $64^{\circ} 36^{\prime} 27.31^{\prime \prime} \mathrm{N}$ & $20^{\circ} 57^{\prime} 04.32^{\prime \prime} \mathrm{E}$ \\
Ireland & $\mathrm{G}$ & $53^{\circ} 35^{\prime} 24.27^{\prime \prime} \mathrm{N}$ & $08^{\circ} 03^{\prime} 33.69^{\prime \prime} \mathrm{W}$ \\
UK & $\mathrm{C}$ & $52^{\circ} 29^{\prime} 17.12^{\prime \prime} \mathrm{N}$ & $00^{\circ} 55^{\prime} 59.12^{\prime \prime} \mathrm{W}$ \\
UK & J & $52^{\circ} 44^{\prime} 34.76^{\prime \prime} \mathrm{N}$ & $01^{\circ} 08^{\prime} 09.87^{\prime \prime} \mathrm{W}$ \\
\hline
\end{tabular}

The eleven genotypes are grouped by six European countries (sample names corresponding to Fig. 1) with latitude and longitude coordinates of their origin

and cross-amplification with other important and closely related forage grass species like $P$. aquatica [16]. Furthermore, microsatellite markers are routinely used to infer invasion routes of invading species [17], and as such could aid in understanding its invasive success in Northern America. Publically available genomic resources for the genus Phalaris are generally scarce; hence the primers are of high value for future research.

\section{Methods}

Total genomic DNA was extracted from eleven genotypes which were collected as part of the European Grass Margins project (Table 1) by either fresh extraction in liquid nitrogen or freeze-drying prior to extraction following a standard cetyltrimethylammonium bromide (CTAB) method [18]. Initially ninety primer pairs were designed using the Primer3 online programme (http://biotools. umassmed.edu/bioapps/primer3_www.cgi) from a normalized EST library consisting of 18,682 P. arundinacea transcripts [19] (sequence data available at the Sequence Read Archive at NCBI, accession number SRP045256) upon searching for microsatellites with Gramene SSRIT (Simple Sequence Repeat Identification Tool; http://archive. gramene.org/db/markers/ssrtool). The following core criteria were applied for primer design: (1) primer melting temperature between 57 and $63{ }^{\circ} \mathrm{C}$ with $60{ }^{\circ} \mathrm{C}$ as optimum; (2) primer size (bp) ranging from 18 to $27{ }^{\circ} \mathrm{C}$ with $20{ }^{\circ} \mathrm{C}$ as optimum; (3) product size (bp) ranging from 100 to a maximum of 400 with 200 as optimum; and (4) CG clump of two. We found microsatellites varying in repeat motifs from di- up to nona-, the majority of which were dinucleotide repeats. The primers used in this study were selected from a range of SSR repeat motifs (Table 2). All primers were synthesised by Metabion international AG and were subsequently tested by PCR on standard agarose gels first. Out of 90 initially tested SSR markers 43 were retained since they produced clear banding pattern in the expected size range on a $3 \%$ MetaPhor $^{\mathrm{TM}}$ agarose gel in $1 \times$ TAE buffer. The further selection process of eight SSR markers which were characterized in detail was based on the indication that they might be highly polymorphic. The PCR products were run on a pre-stained gel with Ethidium bromide and placed at $4{ }^{\circ} \mathrm{C}$ for around $20 \mathrm{~min}$ to aid in obtaining optimal resolution and gel handling characteristics, as per manufacturer's specifications. Primer details and GenBank accessions are provided in Table 3. A template DNA volume of $1 \mu \mathrm{L}(40 \mathrm{ng} / \mu \mathrm{L})$ was amplified with initial denaturation step for $5 \mathrm{~min}$ at $95{ }^{\circ} \mathrm{C}$ followed by 35 cycles each with a denaturation of $30 \mathrm{~s}$ at $95{ }^{\circ} \mathrm{C}, 20 \mathrm{~s}$ at a primer specific annealing temperature, and extension of $20 \mathrm{~s}$ at $72{ }^{\circ} \mathrm{C}$, followed by a final extension at $72{ }^{\circ} \mathrm{C}$ for $7 \mathrm{~min}$. The reaction mixture contained $1 \times$ reaction buffer consisting of $1.25 \mu \mathrm{M}$ dNTPs, $10 \mu \mathrm{M}$ of each primer, and $0.5 \mathrm{U}$ of Taq DNA polymerase (New England BioLabs, Herts, United Kingdom). The primers were tested for polymorphism originating from six European countries, in a total of eleven samples each from a different geographic region (Table 1). Purified PCR products (QIAquick PCR purification kit, Germantown, USA) were then sequenced by Sanger sequencing from both ends by GATC Biotech Ltd., London, England.

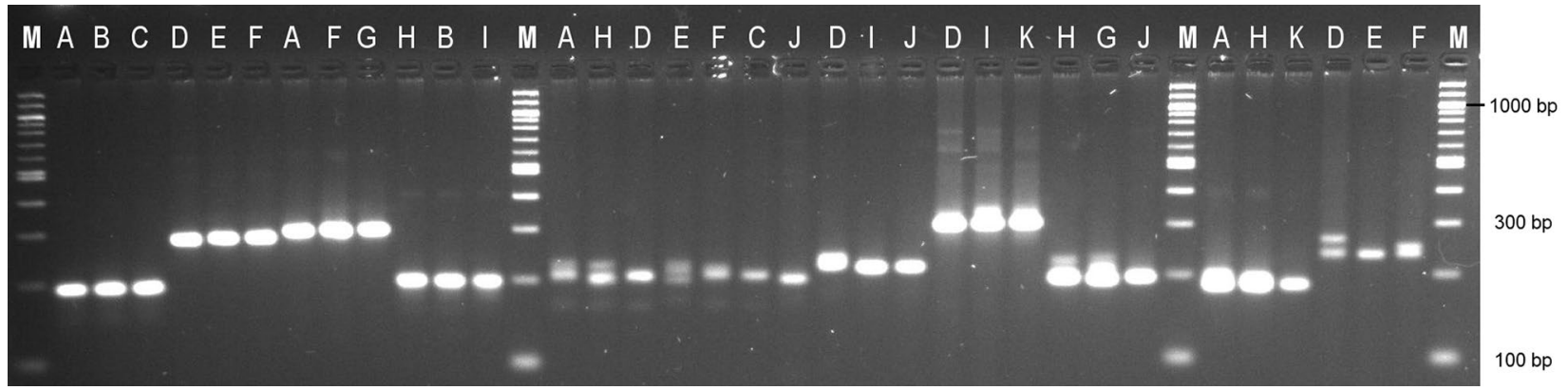

Fig. 1 Representative image of amplified product for ten different EST-SSR markers. The samples were run on $3 \%$ MetaPhor $^{\mathrm{TM}}$ gel and three or, in one case seven different genotypes from wide geographic locations were amplified. The gel was post-stained with $3 \times$ gelRED for 1 h. Lane M-100 bp DNA ladder (New England BioLabs, Herts, United Kingdom); sample order and geographic distribution are defined in Table 1 
Table 2 A collection of forty three successfully amplified primers

\begin{tabular}{|c|c|c|c|c|}
\hline Primer name & Isotiq position & Repeat motif & Primer sequence $\left(5^{\prime}-3^{\prime}\right)$ & $\mathrm{T}_{\mathrm{a}}$ \\
\hline TeaPh_nSSR_1 & 00060-1 & $(\mathrm{AC})_{4}$ & F:TACTTCATTGGGTGGGATGG R: CGCGAATGAAATGAGAAAGC & 54 \\
\hline TeaPh_nSSR_2 & $00060-2$ & $(\mathrm{AT})_{4}$ & F: GGTGGCTAATCTCAGGAATGG R:TGCCCGATAATAAGCACTAGC & 54 \\
\hline TeaPh_nSSR_3 & 08186-1 & $(\mathrm{TTG})_{4}$ & F: GGTAAATTCAGATTATTCCAAAACC R: CCTTTTTGAATGGCAGTTCC & 53 \\
\hline TeaPh_nSSR_4 & 01314-2 & $(\mathrm{TGT})_{4}$ & F: AACGGTGACAAAAGACAAAGC R: CAGCCGTATATCCACAATGC & 54 \\
\hline TeaPh_nSSR_5 & 01313-2 & $(\mathrm{TGT})_{4}$ & F: AACGGTGACAAAAGACAAAGC R: CAGCCGTATATCCACAATGC & 54 \\
\hline TeaPh_nSSR_6 & 08185-3 & $(\mathrm{ATT})_{4}$ & F:TGGCCAACTCTCAGTAGAAGG R: CCATGACCAAAATGAACTCC & 5 \\
\hline TeaPh_nSSR_7 & 00075-2 & $(\mathrm{TTCT})_{4}$ & F:TCCCCTCTTTGGTTTATCATTCG R: GAATCCGGTAAGGTACTTTTGG & \\
\hline TeaPh_nSSR_8 & $00074-2$ & $(\mathrm{TTCT})_{4}$ & F:TCCCCTCTTTGGTTTATCATTCG R: GAATCCGGTAAGGTACTTTTGG & \\
\hline TeaPh_nSSR_9 & 00068-2 & $(\mathrm{TTCT})_{4}$ & F:TCCCCTCTTTGGTTTATCATTCG R: GAATCCGGTAAGGTACTTTTGG & 5 \\
\hline TeaPh_nSSR_10 & $03441-1$ & $(\mathrm{TA})_{4}$ & F:TGCAATGATTTTCTCTATCTTGC R: TCTATCGCTTCACTTTGTCTCG & \\
\hline TeaPh_nSSR_11 & 03440-1 & $(\mathrm{TA})_{4}$ & F:TGCAATGATTTTCTCTATCTTGC R:TCTATCGCTTCACTTTGTCTCG & \\
\hline TeaPh_nSSR_13 & 00265-3 & $(\mathrm{GA})_{4}$ & F: AGCAAGTATGCCGAAAGACC R: GGGAGACCCACACTTACAGC & \\
\hline TeaPh_nSSR_16 & 03439-1 & $(\mathrm{CT})_{4}$ & F: GTACCCGAAACCGACACAGG R: CCCCCATACATGGTCTTACG & \\
\hline TeaPh_nSSR_17 & 03438-1 & $(\mathrm{CT})_{4}$ & F:TTCTCCACGAGGCTCATACC R: GAAGTTACGGGGCTATTTTGC & \\
\hline TeaPh_nSSR_19 & 03440-4 & $(\mathrm{AT})_{4}$ & F:TTCCGAATTAAATGGAGAATCC R: GATAACGGGACATGAAGACTCC & \\
\hline TeaPh_nSSR_20 & $00072-1$ & $(\mathrm{AT})_{4}$ & F: GGTGGCTAATCTCAGGAATGG R:TGCCCGATAATAAGCACTAGC & \\
\hline TeaPh_nSSR_21 & 08188-1 & $(\mathrm{AG})_{4}$ & F: CAATGCCAAAGAAACAATGC R: ACCTCAGATCGAAGCATTCC & \\
\hline TeaPh_nSSR_23 & $00071-1$ & $(\mathrm{AC})_{4}$ & F:TACTTCATTGGGTGGGATGG R: CGCGAATGAAATGAGAAAGC & \\
\hline TeaPh_nSSR_24 & 03440-3 & $(\mathrm{AT})_{5}$ & F: GAATGAAAATGCCAATAAAGTCG R: TTTTAATTTCTCTAATTCGCAAATCC & \\
\hline TeaPh_nSSR_25 & 08351-5 & $(\mathrm{TGC})_{10}$ & F:TCCTATGATCTCTGCCTCAGC R: GCACTGTCCATCAACACACC & \\
\hline TeaPh_nSSR_34 & $01672-8$ & $(\mathrm{CCGAAACA})_{3}$ & F:TTACCGACTCCGTCTTGACC R: GTCGATGGAGATGACGTTGG & \\
\hline TeaPh_nSSR_37 & 03471-10 & $\left(\right.$ TTTTGAA) ${ }_{3}$ & F: GTGTTTGGCCTGTAATCTGG R: CGTAAATGCATCTCTATCTGTTCC & \\
\hline TeaPh_nSSR_42 & 01705-4 & $(\mathrm{GT})_{8}$ & F:TCAAGTGTCATCCGTTGTCC R:TTTTAACGCAAATAGTTTCATCG & \\
\hline TeaPh_nSSR_43 & 02516-1 & $(\mathrm{GT})_{6}$ & F:TGGACTGCACCTAGGAGACC R:TACCACCATGGAACAAAACG & \\
\hline TeaPh_nSSR_45 & $08327-5$ & $(\mathrm{GC})_{4}$ & F: AAAGTACATTGAAAGCTAGTGTCACC R: GCCTCCAAAGCAAGATGC & \\
\hline TeaPh_nSSR_46 & 03588-3 & $(\mathrm{CG})_{6}$ & F:TCTCCGCTCGATCTAAATAGC R:TGTGTGTGCTGAAAGTGTCG & \\
\hline TeaPh_nSSR_47 & 01700-4 & $(\mathrm{CG})_{4}$ & F: GACAGATGGGGCACTACTCC R: GTGTGAGGAATCCACAGTGC & \\
\hline TeaPh_nSSR_48 & 02594-3 & $(\mathrm{TAA})_{6}$ & F: AAGAGTGTCACCATGGAGTGG R: ACCTTCTGAGAGCCTCTTGC & \\
\hline TeaPh_nSSR_49 & 02597-9 & $(\mathrm{GCA})_{5}$ & F: GATACGCTGGAATACCAGAAGG R: GGGAATGGAAACGAACAGG & \\
\hline TeaPh_nSSR_50 & 08302-4 & $(\mathrm{GAA})_{5}$ & F: AAGAGGAAGCCGAAGAGTGG R:TCTGTGGTGCTCAGTTCAGG & \\
\hline TeaPh_nSSR_52 & 08189-10 & $(\mathrm{GAT})_{3}$ & F:TTAACTCGAGGTCATGCATCC R: CCTTTAGCGTCCAAAACTGC & \\
\hline TeaPh_nSSR_54 & $08427-4$ & $(\mathrm{CAA})_{4}$ & F: ACATCCACAGGATTCCATGC R: GCCAGAGATGAGAAGGATGC & \\
\hline TeaPh_nSSR_55 & 02553-8 & $(\mathrm{CAT})_{3}$ & F: AGCAACCAGAACCTGACACG R: AGATGGTACGGCTGGTATGC & \\
\hline TeaPh_nSSR_57 & 02609-7 & $(\mathrm{CGG})_{4}$ & F: GTTCGCTTCGATTTGTTTCC R: CGAAATGAACGGCCTAATCC & \\
\hline TeaPh_nSSR_58 & 08459-13 & $(\mathrm{GCTC})_{4}$ & F:TCCCGACTTCATGAGCTACC R: GGAGGAGCATGTGTGAATGG & \\
\hline TeaPh_nSSR_59 & 00075-2 & $(\mathrm{TTCT})_{4}$ & F:TCCCCTCTTTGTTTATCATTCG R: GAATCCGGTAAGGTACTTTTGG & \\
\hline TeaPh_nSSR_60 & 01318-11 & $(\mathrm{AGGA})_{3}$ & F: GGGCTTTCTACATAGGGATCG R:TTGATCTTTACGGTGCTTTCC & \\
\hline TeaPh_nSSR_65 & 08352-6 & $(\mathrm{AGG})_{4}$ & F: CTCCACCACCTCCACAAAAT R:TTTCGTCTTTGTGCTTGCTG & \\
\hline TeaPh_nSSR_66 & 00769-7 & $(\mathrm{TTG})_{3}$ & F: CGTTGTGCCTTAGCTACTTGC R: ATGATCCAACCAGCTTGACC & \\
\hline TeaPh_nSSR_70 & 08235-11 & $(\mathrm{TGCT})_{3}$ & F: CCTTGAGGAGGATGATGTGG R:TCCTGATGTGCTTGATGAGC & \\
\hline TeaPh_nSSR_71 & $08212-11$ & $(\mathrm{TTCA})_{3}$ & F: GATGGAATCACGCTCTGTAGG R: GGGCAGTAGCGAAGAGATCC & \\
\hline TeaPh_nSSR_80 & 03674-1 & $(\mathrm{GAT})_{4}$ & F: CCAAACCCAGTTGTGACTCC R: GGCATCAGAATCATAGTCATCG & \\
\hline TeaPh_nSSR_81 & $03659-2$ & $(\mathrm{GGT})_{5}$ & F: CGGTTGGACTGATAACATTGG R: CCCATCCTGAGTCGTCACC & \\
\hline
\end{tabular}

Forty three primers that successfully amplified a distinct band of expected size on a $3 \%$ MetaPhor agarose gel, their Isotiq position on the EST assembly, repeat motif, forward and reverse primer sequences $\left(5^{\prime}-3^{\prime}\right)$ and annealing temperature $(\mathrm{Ta})$

\section{Results and discussion}

43 out of the 90 initially designed microsatellite marker primer pairs proved to amplify successfully with discreet bands. Eight highly polymorphic SSR loci for $P$. arundinacea were identified which ranged in size from around 300 to $200 \mathrm{bp}$ in length (Table 3). Sanger sequencing revealed 
Table 3 Eight highly polymorphic nuclear EST-SSR markers with GenBank accession numbers and size range

\begin{tabular}{ll}
\hline Primer name & Clone; GenBank accession no. \\
\hline TeaPh_nSSR_2 & KU316389; KU316392; KU316395 \\
TeaPh_nSSR_4 & KU316399; KU316400; KU316403; KU316416; KU316414 \\
TeaPh_nSSR_7 & KU316423; KU316427; KU316426; KU316429; KU316433 \\
TeaPh_nSSR_24 & KU316439; KU316441; KU316443; KU316444 \\
TeaPh_nSSR_46 & KU316451; KU316452; KU316458; KU316467; KU316471 \\
TeaPh_nSSR_47 & KU316485; KU316486; KU316489; KU316476 \\
TeaPh_nSSR_49 & KU316491; KU316492; KU316495; KU316498 \\
TeaPh_nSSR_80 & KU316500; KU316501; KU316506; KU316512 \\
\hline
\end{tabular}

The study developed eight polymorphic EST-SSR markers containing polymorphism such as SSRs, InDels and SNPs

a

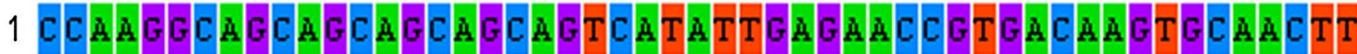

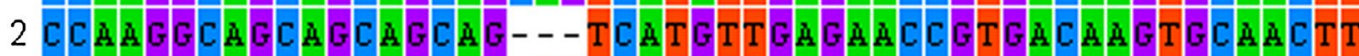

3 ССС д GGCh

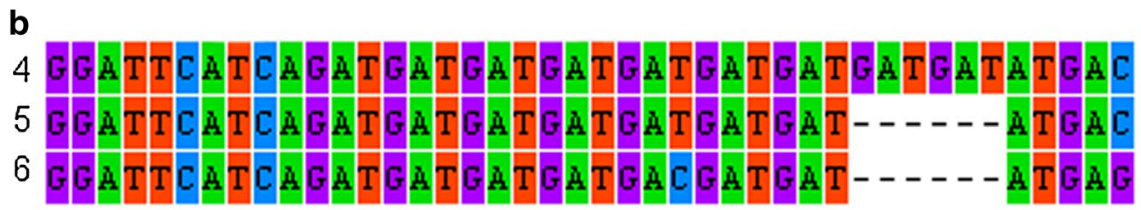

C

7 CGGACTTCGGAGGAGAGGCCGCGCGCGCGC--CGGCGTCGGGGGCGGG

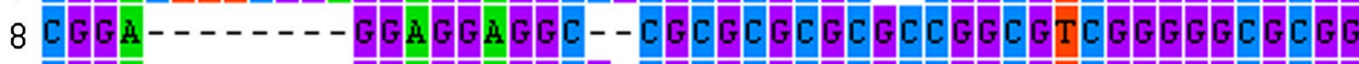

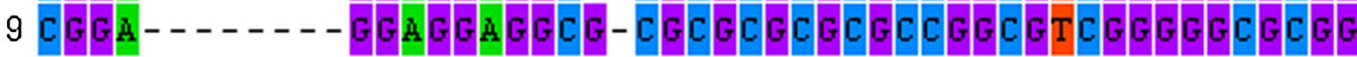

Fig. 2 Example of motif changes, indels and SNPs in the microsatellite region of nine representative samples. a fragments amplified with primer TeaPh_nSSR49; 1 Poland (accession number KU316491); 2 Ireland (accession number KU316495); 3 Ireland (accession number KU316498). b fragments amplified with primer TeaPh_nSSR80; 4 Poland (accession number KU316501); 5 Poland (KU316502); 6 Poland (KU316514). c fragments amplified with primer TeaPh_nSSR46; 7 Germany (accession number KU316457); 8 Denmark (KU316467); 9 United Kingdom (KU316471)

numerous single nucleotide polymorphisms (SNPs), motif changes as well as indels in the genotypes of Phalaris from the different geographical locations (Fig. 2). Changes in the motif length varied from tetra- to tri-, di- and even mononucleotide repeats. In some instances the repeat motif was longer than expected (from tetrato hepta- and nonanucleotide repeats). Changes were also observed from penta- to tetra- and hexanucleotide repeats. These microsatellite markers are useful for studying genetic diversity and population structure as well as for elucidation of $P$. arundinacea invasive status. As an increasingly important energy crop and well established forage crop species, the improvement of bioenergy and palatability traits for livestock, $P$. arundinacea might be of interest to breeders worldwide. The markers can be also further cross-amplified in closely related taxa like
P. aquatica, an important forage species in Australasia, and other members of the genus Phalaris like $P$. minor a widely spread grass weed. These eight microsatellite markers will be of interest and value in addressing taxonomic and biogeographic issues because of the samples' wide geographic distribution.

\section{Authors' contributions}

SB, MK, TS, TV and TRH conceived the study. SB, MJ, MK, TV and TRH wrote the manuscript. SB, MK, MJ and TV conducted the experiments. All authors read and approved the final manuscript.

\section{Author details}

${ }^{1}$ Teagasc Crops Environment and Land Use Programme, Oak Park Research Centre, Carlow, Ireland. ${ }^{2}$ Department of Botany, School of Natural Sciences, Trinity College Dublin, Dublin, Ireland.

\section{Acknowledgements}

We are grateful to Yasmin Soppa and Niall McLoughlin for technical help in the lab. 


\section{Competing interests}

The authors declare that they have any competing interests.

\section{Funding}

This study has been financed under the collaborative European Community Framework FP7 project GrassMargins (KBBE-2011-5-289461).

Received: 1 February 2016 Accepted: 16 March 2016

Published online: 22 March 2016

\section{References}

1. Smulders MJM, Esselink GD, Everaert I, De Riek J, Vosman B. Characterisation of sugar beet (Beta vulgaris L. ssp. vulgaris) varieties using microsatellite markers. BMC Genet. 2010;11:41.

2. Turchetto C, Segatto ALA, Beduschi J, Bonatoo SL, Freitas LB. Genetic differentiation and hybrid identification using microsatellite markers in closely related wild species. AoB Plants. 2015;7:1-12.

3. Chase M, Kesseli R, Bawa K. Microsatellite markers for conservation and population genetics of tropical tree species. AJB. 1996;83:51-7.

4. Forster JW, Hand ML, Cogan NOI, Hayes BJ, German C, Smith KF. Resources and strategies for implementation of genomic selection in breeding of forage species. Crop and Pasture. Science. 2014;65:1238-47.

5. Sheaffer CC, Marten GC. Reed canarygrass: An introduction to grassland agriculture. In: Barnes RF, Miller RF, Darrell A, Nelson CJ, editors. Forages. Iowa: IOWA State University Press; 1995. p. 335-43.

6. Ghica A, Samfira I. Bibliographic study of genetic process in Phalaris arundinacea. Res J Agric Sci. 2011;43:65-71.

7. Apfelbaum SI, Sams CE. Ecology and control of reed canary grass (Phalaris arundinacea L.). Nat Areas J. 1987;7:69-74.

8. Lavergne S, Molofsky J. Increased genetic variation and evolutionary potential drive the success of an invasive grass. Proc Natl Acad Sci. 2007; 104:3883-8.
9. Martina JP, Von Ende CN. Increased spatial dominance in high nitrogen, saturated soil due to clonal architecture plasticity of the invasive wetland plant, Phalaris arundinacea. Plant Ecol. 2013;214:1443-53.

10. Ramstein GP, Lipka AE, Lu F, Costich DE, Cherney JH, Buckler ES, Casler MD. Genome-wide association study based on multiple imputation with low-depth sequencing data: application to biofuel traits in reed canarygrass. G3: Genes |Genomes |Genetics. 2015. http://www.g3journal. org/content/early/2015/03/12/g3.115.017533.full.pdf+html. Accessed 13 July 2015.

11. Rydberg PA. Phyotogeographical notes on the Rocky Mountain region. V. Grasslands of the subalpine and montane zones. Bull Torrey Bot Club. 1915;42:629-42.

12. Brodersen C, Lavergne S, Molofsky J. Genetic variation in photosynthetic characteristics among invasive and native populations of reed canarygrass (Phalaris arundinacea). Biol Invasions. 2008;10:1317-25.

13. Picard $C R$, Fraser $L H$, Steer $D$. The interacting effects of temperature and plant community type on nutrient removal in wetland microcosms. Bioresour Technol. 2005;96:1039-47.

14. Rice J, Pinkerton B. Reed canarygrass survival under cyclic inundiation. J Soil Water Conserv. 1993:48:132-5

15. Pakhala K, Pihala M. Different plant parts as raw material for fuel and pulp production. Ind Crops Prod. 2000;11:119-28.

16. Carlson IT, Oram RN, Suprenant J. Reed canarygrass and other Phalaris species. Agron Monogr. 1996;34:569-604.

17. Estoup A, Guillemaud T. Reconstructing routes of invasion using genetic data: Why, how and so what? Mol Ecol. 2010;19:4113-30.

18. Doyle JJ, Doyle JL. Isolation of plant DNA from fresh tissue. Focus. 1990;12:13-5.

19. Haiminen N, Klaas M, Zhou Z, Utro F, Cormican P, Didion T, Jensen CS, Mason C, Barth S, Parida L. Comparative exomics of Phalaris cultivars under salt stress. BMC Genom. 2014;15(Suppl 6):S18.

\section{Submit your next manuscript to BioMed Central and we will help you at every step:}

- We accept pre-submission inquiries

- Our selector tool helps you to find the most relevant journal

- We provide round the clock customer support

- Convenient online submission

- Thorough peer review

- Inclusion in PubMed and all major indexing services

- Maximum visibility for your research

Submit your manuscript at www.biomedcentral.com/submit
(O) BioMed Central 